\title{
Stratégies d'adaptation à la réduction des services écosystémiques : cas des potentialités de substitution de trois espèces forestières dans le Sud-Ouest du Burkina Faso
}

\author{
Sibiry Albert KABORÉ ${ }^{1 *}$, Katharina SCHUMANN ${ }^{2}$, Mipro HIEN ${ }^{1}$, \\ Anne Mette LYKKE $^{3}$, Karen HAHN ${ }^{2}$ et Hassan Bismarck NACRO ${ }^{1}$ \\ ${ }^{I}$ Institut du Développement Rural, Université Polytechnique de Bobo-Dioulasso, 01 BP 1091 Bobo-Dioulasso \\ 01, Burkina Faso. \\ ${ }^{2}$ Department of Ecology and Geobotany, Institute for Ecology, Evolution and Diversity, Johann Wolfgang \\ Goethe University, Max von Laue Str. 13, PO Box 19, 60438 Frankfurt am Main, Germany. \\ ${ }^{3}$ Department of Bioscience, Aarhus University, Vejlsфvej 25, 8600 Silkeborg, Denmark. \\ *Auteur correspondant : E-mail : kaborealbert64@yahoo.fr; Tel :00226 70164914
}

\section{RESUME}

Les conséquences de la perte de biodiversité sont entre autres la baisse des services écosystémiques, avec des incidences graves sur la santé des populations, leur alimentation, voire leur habitat. Dans ce contexte, les populations locales ont tendance à adopter des stratégies d'adaptation qu'il importe d'identifier et d'en analyser la durabilité. L'objectif de cette l'étude était de déterminer les substituts de trois plantes (Crateva adansonii D.C., Sarcocephalus latifolius (Smith) Buce et Burkea africana Hook.) à haute valeur sociale et culturelle dans le Sud-Ouest du Burkina Faso. Il s'agit de fournir des informations à même d'orienter les mesures de conservation et d'anticipation des effets de la déforestation. Une enquête a été conduite auprès de 253 personnes de neuf localités appartenant à trois groupes ethniques. Les résultats ont montré que les populations ont identifié seize substituts pour $C$. adansonii, vingt-deux pour $S$. latifoliius et seize pour $B$. africana. La plupart des substituts sont des espèces locales ou exotiques, et très peu de produits manufacturés. Il est donc possible, plutôt que d'interdire sans proposition de rechange à l'exploitation des espèces menacées, d'inverser la tendance en accompagnant les populations à utiliser les diverses potentialités de substitution des espèces. Ceci est un axe de conservation efficace des ressources végétales.

() 2015 International Formulae Group. All rights reserved.

Mots clés : Crateva adansonii D.C, Sarcocephalus latifoliius (Smith) Buce, Burkea africana Hook., biodiversité, conservation

\section{INTRODUCTION}

L'histoire de la civilisation a été de tout temps pour l'homme une recherche permanente de ressources naturelles (Fisher et al., 2009). De par le monde, plusieurs auteurs s'accordent à reconnaitre que les services des écosystèmes tendent à diminuer drastiquement (Dobson et al., 2006 ; Limoges, 2009). Face à la dégradation continue de l'environnement, plusieurs stratégies ont été développées pour 
une meilleure protection de la biodiversité (Mawdsley et al., 2006).

Malgré les nombreuses mesures de conservation des formations adoptées dans les pays en voie de développement, la perte de biodiversité liée aux activités humaines se fait toujours à une ampleur et à un rythme inquiétant (Konaté et Linsenmair, 2010). Les consequences resultants du changement de la structure de la végétation ont conduit les populations à adopter differentes alternatives à la réduction des biens et services écosystemiques. Le volet substituts des plantes a été peu abordé dans les études traitant des utilisations des plantes. Cependant, pour pallier à la rarefaction de certaines espèces, d'autres études ont montré que les populations optent, soit pour un remplacement d'une plante par une autre (Monteiro et al., 2006), soit pour l'utilisation d'une partie à la place d'une autre partie de la même plante (Zschocke et al., 2000). Cette stratégie s'avère être une bonne approche pour la conservation des espèces.

La notion de substitution des espèces doit être comprise sous l'angle de remplacement, en vue de rendre totalement ou presque le même service. Une espèce est substituée par une autre quand la plante substituée n'est pas à la portée de son utilisateur. La substitution peut aussi découler d'un processus d'amélioration ou de recherche de produits plus disponibles et moins coûteux, et qui donnent les mêmes résultats voire de meilleurs résultats. C'est le cas, au niveau industriel, de la substitution du cacao par le beurre de karité dans les chocolateries (Bayala et al., 2009).

Cette étude a porté sur Crateva adansonii, Sarcocephalus latifolius et Burkea africana qui sont des espèces à haute valeur sociale et culturelle pour la population du Sud-Ouest du Burkina Faso. Ces espèces ont disparues ou sont ménacées dans certaines regions du pays (Hahn-Hadjali et Thiombiano, 2000). Les feuilles de $C$. adansonii sont très consommées mais l'espèce est devenue rare en peuplement naturel dans le Sud-Ouest. Les racines de $S$. latifolius y sont exploitées à des fins thérapeutiques (Kaboré et al., 2014). Le commerce des racines, les modes de récolte très destructifs par déracinement complet des pieds et le défrichage des zones bien drainnées où affectionne la plante ont accentué le recul de ses peuplements. Quant à B. africana, son bois est reconnu très dur et très résistant aux attaques des termites et est de ce fait beaucoup utilisé dans la construction des maisons traditionnnelles. Ces dernières sont reputées être très consomatrices en bois. L'utilisation de l'entiereté du tronc des arbres conjuguée à la forte demande en bois d'œuvre a contribué à la baisse de la disponilité de cette espèce.

La question principale qui se pose et à laquelle cette étude a tenté de répondre est la suivante: face au recul des peuplements des trois espèces, quelles sont les stratégies des populations locales en termes de substitution aux manques de leur produits? La présente étude vise donc à identifier les substituts des trois espèces. Son objectif était de voir si la partie de la plante utilisée est remplacée, soit part un autre organe de la même plante ou tout autre organe d'une autre plante ou soit par un produit manufacturé. Le travail a aussi consisté à vérifier si le choix des substituts dépend de la localité, du sexe ou du groupe ethnique des personnes enquêtées. Ce travail vise à orienter les mesures de conservation qui prennent en compte les stratégies d'adaptation des populations locales.

\section{MATERIEL ET MÉTHODES \\ Zone d'étude}

L'étude a été conduite dans la région du Sud-Ouest du Burkina Faso (Figure 1). Les travaux ont été menés au sein de trois groupes ethniques que sont les "Lobi", les "Dagara" et les "Birifor". Trois villages par groupe ethnique ont été choisis à cet effet. Ce sont: Bombara $\left(10^{\circ} 47 \mathrm{~N}\right.$ et $\left.3^{\circ} 16^{\prime} \mathrm{O}\right)$, Tioyo (10 $38^{\circ}$ $\mathrm{N}$ et $\left.3^{\circ} 13^{\prime} \mathrm{O}\right)$ et Tinguera $\left(10^{\circ} 49 \mathrm{~N}\right.$ et $3^{\circ} 22^{\prime}$ O) pour les "Lobi", Djikoloko $\left(10^{\circ} 56 \mathrm{~N}\right.$ et $\left.3^{\circ} 07 \mathrm{O}\right)$, Bontioli $\left(10^{\circ} 51 \mathrm{~N}\right.$ et $\left.3^{\circ} 00^{\prime} \mathrm{O}\right)$ et Zambo $\left(10^{\circ} 49^{\prime} \mathrm{N}\right.$ et $\left.2^{\circ} 58^{\prime} \mathrm{O}\right)$ pour les “Dagara" et Mezan (9 $9^{\circ} 2^{\prime} \mathrm{N}$ et $\left.2^{\circ} 56^{\prime} \mathrm{O}\right)$, Titi 
$\left(9^{\circ} 53^{\prime} \mathrm{N}\right.$ et $\left.2^{\circ} 56^{\prime} \mathrm{O}\right)$ et Tamipar $\left(9^{\circ} 49^{\prime} \mathrm{N}\right.$ et $2^{\circ} 44$ ' O) pour les "Birifor".

La région du Sud-Ouest appartient à la zone sud soudanienne caractérisée par l'espèce grégaire Isoberlinia doka (Boussim, 2010) à laquelle s'associent Vitellaria paradoxa et des espèces appartenant principalement aux Césalpiniacées, Combrétacées, Rutacées et Mimosacées. Les savanes arborées et arbustives sont les principaux types de végétation.

A Gaoua $\left(10^{\circ} 20^{\prime} \mathrm{N}\right.$ et $\left.3^{\circ} 10^{\prime} \mathrm{O}\right)$ dans le centre de la région du Sud-Ouest, la pluviosité annuelle varie entre $900 \mathrm{~mm}$ et $1300 \mathrm{~mm}$ avec une moyenne de $1000 \mathrm{~mm}$. La température moyenne annuelle est de $20{ }^{\circ} \mathrm{C}$ pour le minima et $34{ }^{\circ} \mathrm{C}$ pour le maxima (INSD, 2009). L'altidude moyenne de la région du Sud-Ouest oscille autour de $260 \mathrm{~m}$. On y trouve deux saisons distinctes, une pluvieuse de juin à septembre et une sèche d'octobre à mai. Les populations vivent principalement de l'agriculture extensive et de l'exploitation forestière, ce qui accroît les pressions sur les ressources biologiques. En outre, des études ont montré que le Sud du Burkina Faso est la partie qui sera fortement et négativement affectée par les changements climatiques en terme de perte de biodiversité végétale (Heubes et al., 2013).

\section{Présentation des espèces étudiées}

Crateva adansonii D.C. (synonyme: Crataeva religiosa) appartient à la famille des Capparaceae. A maturité, la plante mesure 10 $\mathrm{m}$ de haut et porte une écorce lisse. Ses feuilles sont trifoliolées, alternes et regroupées en touffes terminales sur les rameaux. Son inflorescence est un racème, à pétales blanches avec des étamines et un pistil rouge violet. Son fruit est une baie de 4 à $8 \mathrm{~cm}$ de diamètre. Son aire de distribution s'étend du Sénégal à l'Erythrée. Elle est retrouvée dans les zones sahéliennes et soudaniennes, sur les sols légers, aux abords des cours d'eau (Arbonnier, 2009). Ses feuilles sont très consommées en sauce dans le Sud-Ouest du Burkina Faso.
Sarcocephalus latifolius (Smith) Buce (synonyme : Nauclea latifolia) est un arbre ou arbuste de la famille des Rubiaceae présente dans les galeries forestières et les savanes soudano-guinéennes sur les sols bien drainés. Elle mesure généralement 4 à $5 \mathrm{~m}$ de hauteur. Ses feuilles sont simples et luisantes (Arbonnier, 2009). Son fruit est une baie rouge marron. C'est une plante médicinale reconnue dans la pharmacopée traditionnelle. Elle présente des intérêts thérapeutiques qui ont fait l'objet de nombreux travaux (Amos et al., 2005 ; Iwueke et Nwodo, 2008 ; Yesufu et al., 2010; Boumendjel et al., 2013). L'utilisation de ses racines contre les maux de ventre constitue sa première utilisation dans le Sud-Ouest du Burkina Faso (Kaboré et al., 2014).

Burkea africana Hook. est une espèce de la famille des Caesalpiniaceae. C'est un arbre de 10-12 $\mathrm{m}$ de haut vivant dans les savanes soudaniennes et guinéennes. Elle est retrouvée du Sénégal jusqu'en Afrique Orientale. Ses feuilles sont alternes. Son inflorescence est un fascicule terminal qui donne des gousses indéhiscentes (Arbonnier, 2009). L'espèce affectionne les hautes altitudes. Son bois est dur et résistant aux attaques des termites (Neya et al., 2004 ; Yaméogo et al., 2013). Sa forte dureté s'expliquerait par le caractère hydrophobe du bois et sa forte stabilité dimensionnelle (Neya et al., 2004). L'espèce est très sollicitée dans la construction des maisons traditionnelles chez les trois groupes ethniques.

\section{Collecte et analyse des données}

Une pré-enquête sur les possibilités de substitution des biens fournis par ces trois espèces a d'abord été ménée dans le village de Bamako $\left(10^{\circ} 55^{\prime} \mathrm{N}\right.$ et $3^{\circ} 19^{\prime} \mathrm{O}$, Sud-Ouest du Burkina Faso) auprès de 30 personnes. L'ojectif a été de voir la faisabilité de l'étude et de cerner les difficultés dans la formulation des questions.

Un échantillon de 253 personnes dont 128 hommes et 125 femmes ont ensuite accepté de participer à l'enquête proprement 
dite. Les enquêtés ont été individuellement interviewés. Ils ont été choisis sur la base de leurs connaissances des espèces et sur leur consentement. L'étude a concerné 30 individus par village, excepté celui de Tamipar où il n'y avait pas autant de personnes qui remplissaient les conditions de l'enquête. Toutefois, l'équilibre numérique entre les sexes a été respecté dans toutes les localités.

Un exposé de la problématique de la baisse des peuplements, et un rappel des principaux usages des trois plantes (feuilles comme nourriture pour $C$. adansonii, racines comme médicament pour $S$. latifolius et bois d'œuvre pour B. africana) a d'abord été fait à l'enquêté. Il est chaque fois précisé à l'enquêté que le substitut peut être issu d'une plante ou être un produit manufacturé.

Seuls les substituts cités par au moins deux enquêtés ont été retenus. Une analyse en composantes principales (ACP) a été utilisée. Le but de cette analyse est de révéler l'existence de groupes de personnes qui pourront avoir les mêmes préférences (Heubach et al., 2013) pour les mêmes substituts en considérant leur appartenance aux différents villages, groupes ethniques et sexes. Les données ont été traitées avec les logiciel PC-ORD 6.0 (McCune et Mefford, 2011) et Excel 2007.

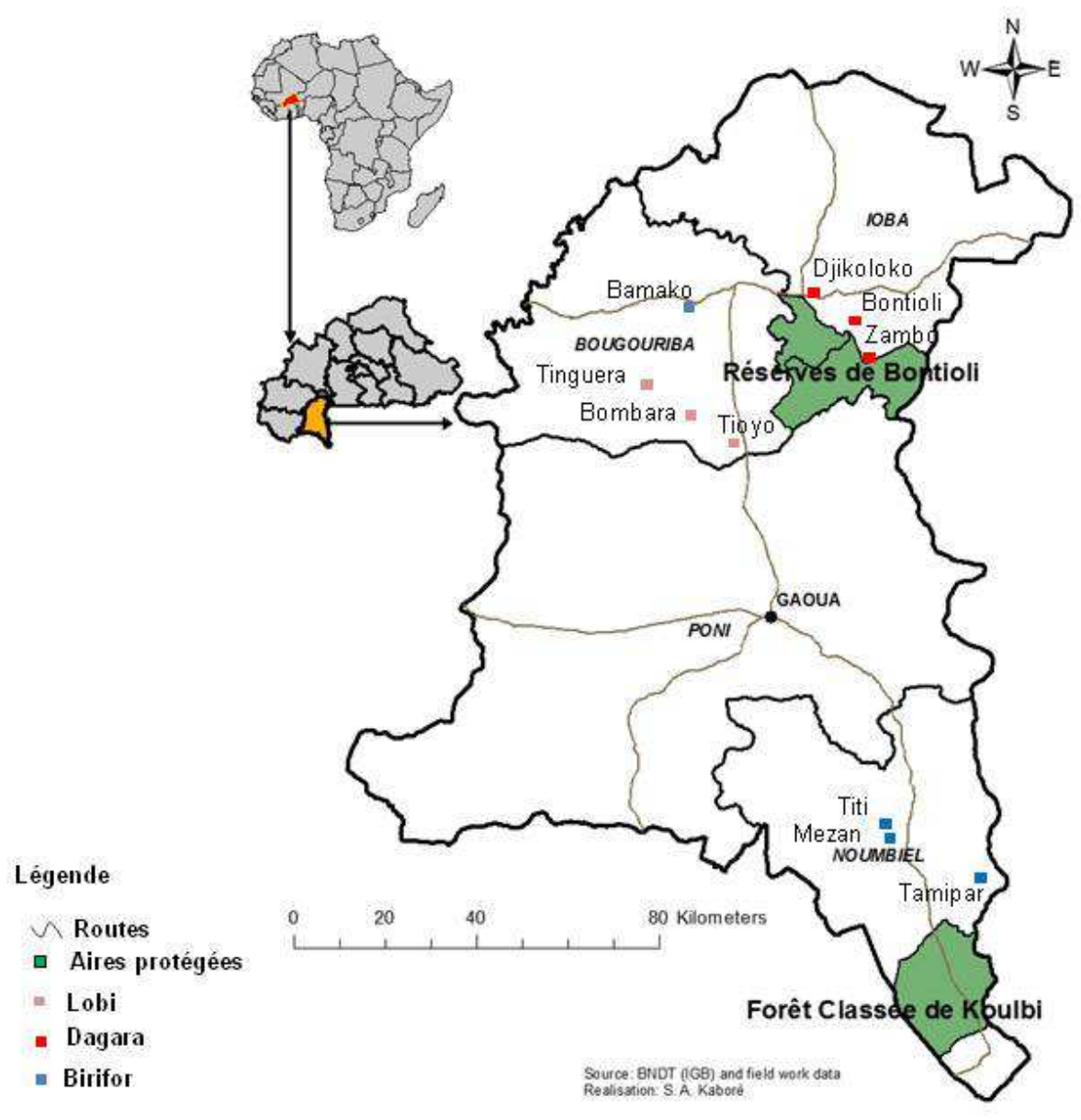

Figure 1 : Localisation de la zone d'étude. 


\section{RESULTATS}

Substituts de Crateva adansonii D.C.

Quinze espèces à feuilles comestibles ont été rapportées comme substituts de $C$. adansonii. Les deux premières espèces, Moringa oleifera et Vitex doniana, sont connues de tous les groupes ethniques (Figure 2). Parmi les espèces, trois sont des herbacées (Hibiscus asper, Ceratotheca sesamoides et Cassia tora) et une est une liane (Leptadenia hastata). En plus de ces espèces, un certain nombre de plantes (choux, aubergine, etc.) regroupées sous le terme légumes a également été mentionné. Les feuilles sont les principaux organes utilisés. Tous les substituts sont des plantes locales récoltées dans leurs milieux naturels. Seul Moringa oleifera et les légumes sont plantés (Tableau 1). Les résultats montrent toutefois qu'une faible proportion des enquêtés (3,3\%) n'a pas trouvé de substitut aux feuilles de $C$. adansonii.

Les "Lobi" optent pour Moringa oleifera, Vitex doniana, Adansonia digitata et Strychnos spinosa. Les "Dagara" ont tendance à plus préférer Moringa oleifera, Vitex doniana, Adansonia digitata, Strychnos spinosa et Balanites aegyptiaca comme substituts pendant que les "Birifor" optent plus pour Moringa oleifera, Vitex doniana, Adansonia digitata, Pterocarpus santalinoides et Ceiba pentandra (Figure 2).

Les analyses en composantes principales n'ont pas mis en évidence un regroupement des enquêtés selon la localité ou le sexe. On aboutit par contre à un groupement des enquêtés selon les groupes ethniques. Le premier et le second axe expliquent respectivement $35,08 \%$ et $22,47 \%$ de la variance des données (Figure 3 ).

\section{Substituts de Sarcocephalus latifolius (Smith) Buce}

Vingt-deux substituts ont été rapportés pour cette espèce. Cassia sieberiana, connue de toutes les ethnies, est la première plante utilisée contre les maux de ventre en remplacement de $S$. latifolius. Elle est suivie de Strychnos spinosa et de Burkea africana. Ces deux substituts sont connus des "Birifor" mais sont presque ignorés des autres ethnies (Figure 4). Les médicaments conventionnels $\left(6^{\text {ième }}\right.$ substitut $)$ et traditionnels $\left(17^{\text {ième }}\right.$ substitut) ont été également mentionnés. Les racines constituent les organes les plus utilisés. Les espèces de remplacement sont essentiellement des plantes locales à l'exception de Mangifera indica et de Moringa oleifera (Tableau 2).

Près de $9,1 \%$ des personnes interrogées n'ont trouvé aucun substitut aux racines de $S$. latifolius. Les "Lobi" optent pour Cassia sieberiana, Ziziphus mucronata et Terminalia laxiflora. Les "Dagara" penchent plus pour Cassia sieberiana, les médicaments modernes, Terminalia laxiflora et Securidaca longepedunculata. Les "Birifor" préfèrent Cassia sieberiana, Strychnos spinosa, Burkea africana, Vernonia colorata et Pseudocedrela kotschyi.

Les analyses en composantes principales ont mis en évidence un regroupement des enquêtés selon l'appartenance au groupe ethnique et à la localité mais une absence de regroupement pour le sexe. Le premier et le second axe expliquent respectivement $19,1 \%$ et $15,4 \%$ de la variance des données (Figure 5).

\section{Substituts de Burkea africana Hook.}

Seize espèces ont été rapportées comme substituts au bois de B. africana pour la construction. Pterocarpus erinaceus et Anogessus leiocarpa sont les substituts de premier choix (Figure 6). Certains substituts comme Prosopis africana et Detarium microcarpum sont utilisés presque exclusivement par les "Lobi" et les "Birifor" respectivement (Figure 6). Seules trois 
espèces que sont Azadirachta indica, Eucalyptus camadulensis et Tectona grandis sont plantées dans la zone d'étude (Tableau 3). Environ $3 \%$ des enquêtés n'ont cependant pas trouvé de substitut au bois de B. africana.

Les "Lobi" ont une préférence pour Pterocarpus erinaceus, Anogeissus leiocarpa, Prosopis africana, Vitellaria paradoxa et Azadirachta indica. Les "Dagara" utilisent plutôt Pterocarpus erinaceus, Anogeissus leiocarpa, Vitellaria paradoxa et Terminalia laxiflora. Les "Birifor" choisissent Pterocarpus erinaceus, Anogeissus leiocarpa, Vitellaria paradoxa et Detarium microcarpum (Figure 6).

Les analyses en composantes principales ont uniquement mis en évidence un regroupement des enquêtés selon le groupe ethnique. Le premier et le second axe expliquent respectivement $22,8 \%$ et $26,8 \%$ de la variance des données (Figure 7).

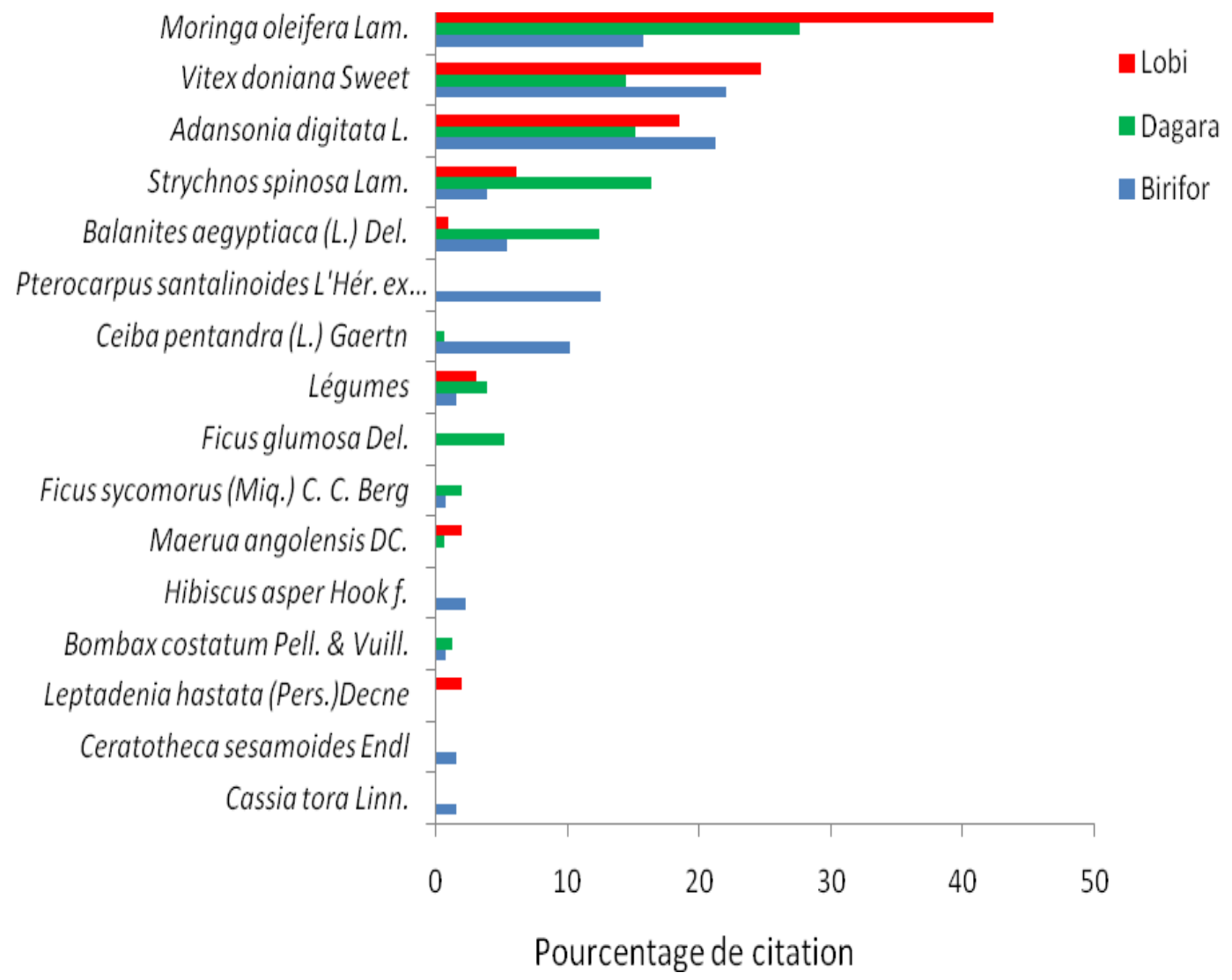

Figure 2: Substituts de Crateva adansonii selon les groupes ethniques dans le Sud-Ouest du Burkina Faso. 


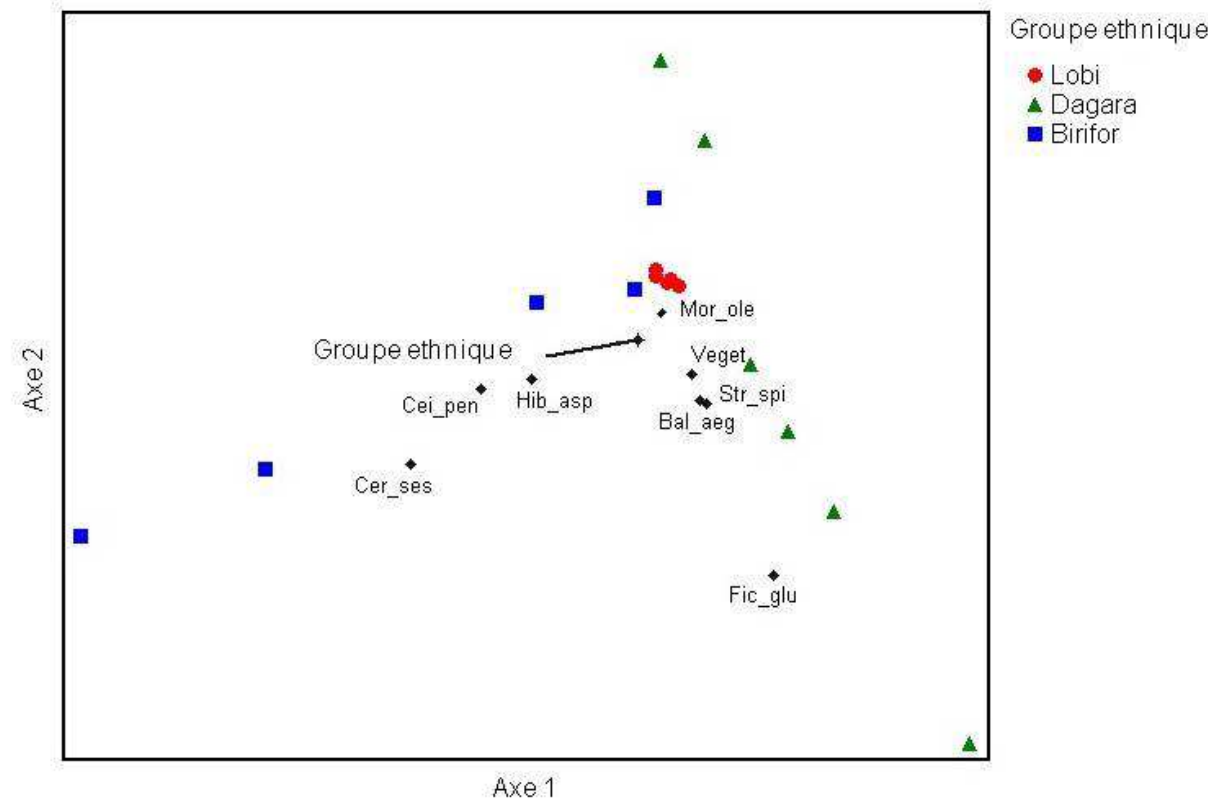

Figure 3 : Ordination des substituts de Crateva adansonii. Mor_ole : Moringa oleifera, Veget : légumes, Cei_pen : Ceiba pentandra, Hib_asp : Hibiscus asper, Str_spi : Strychnos spinosa, Bal_aeg : Balanites aegyptiaca, Cer_ses : Ceratotheca sesamoides, Fic_glu : Ficus glumosa

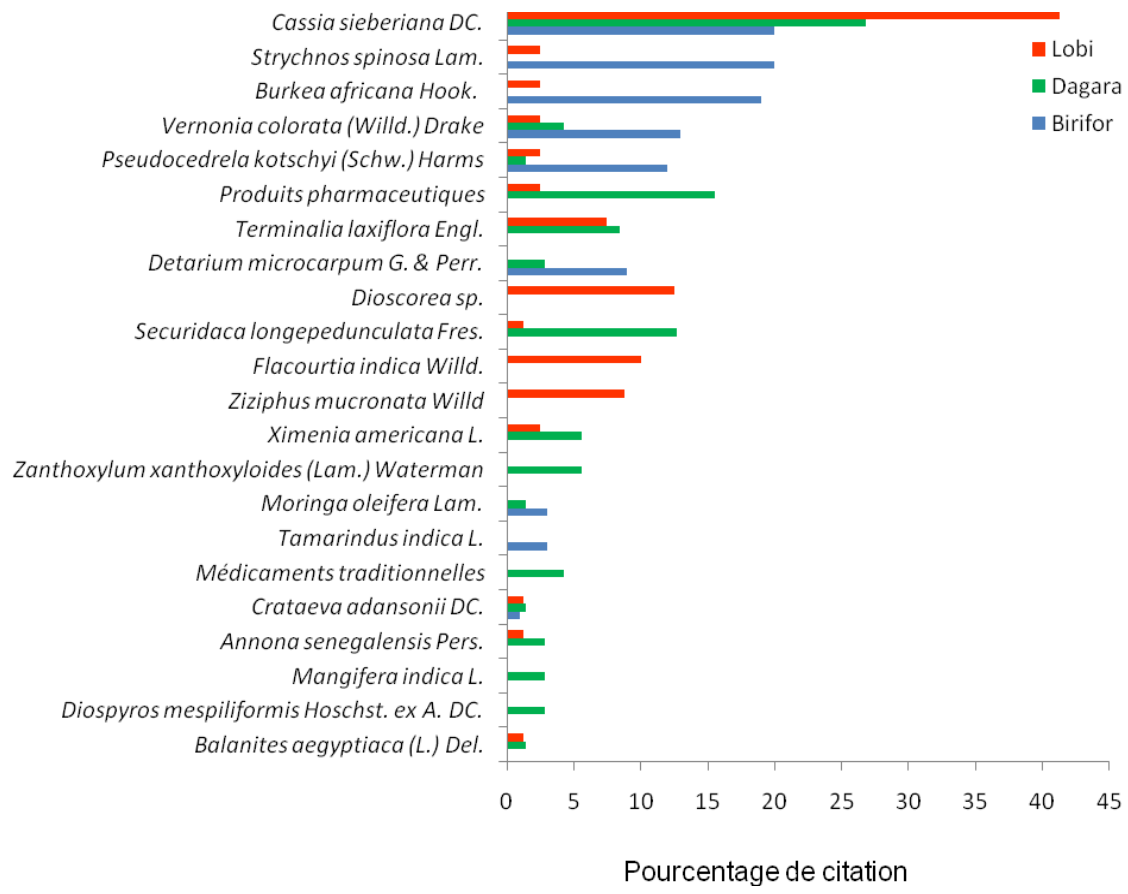

Figure 4 : Substituts de Sarcocephalus latifolius selon les groupes ethniques dans le Sud-Ouest du Burkina Faso. 


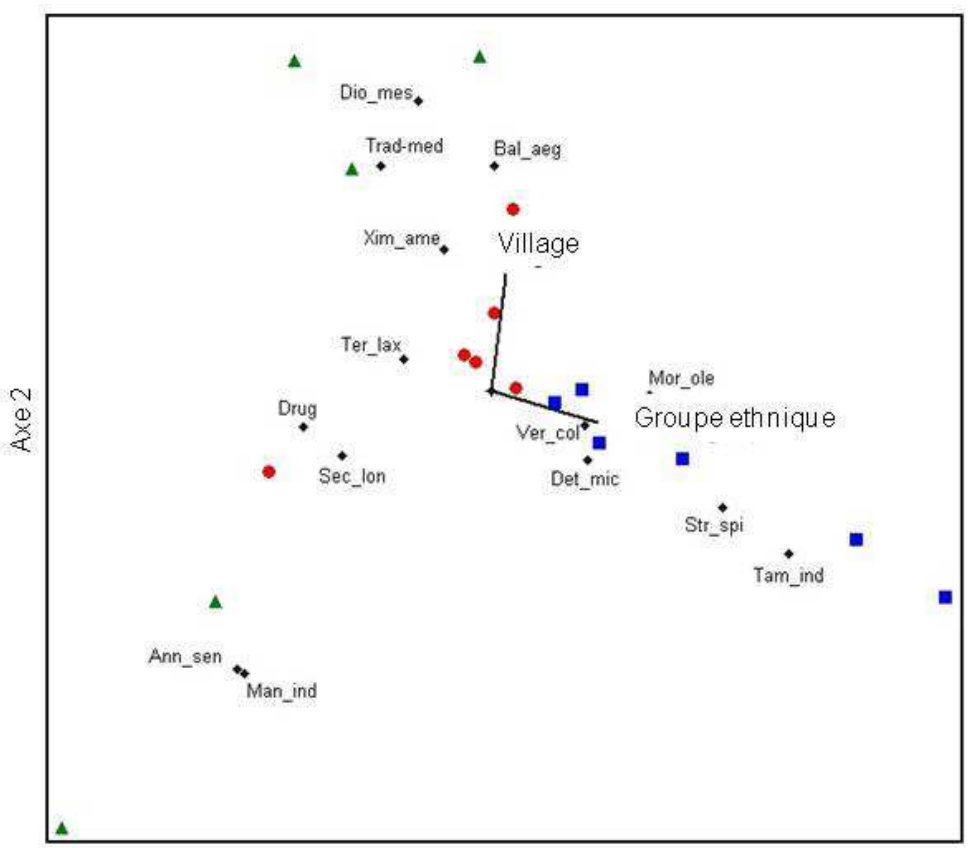

Groupe ethnique

- Lobi

A Dagara

- Birifor

Axe1

Figure 5 : Ordination des substituts de Sarcocephalus latifolius. Ann_sen : Annona senegalensis, Bal_aeg: Balanites aegyptiaca, Det_mic : Detarium microcarpum, Dio_mes : Diospyros mespiliformis, Man_ind : Mangifera indica, Mor_ole: Moringa oleifera, Sec_lon: Securidaca longepedunculata, Str_spi: Strychnos spinosa, Tam_ind: Tamarindus indica, Ter_lax: Terminalia laxiflora, Ver_col: Vernonia colorata, Xim_ame : Ximenia americana, Drug : produits pharmaceutiques, Tra-med : produits de la médecine traditionnelle.

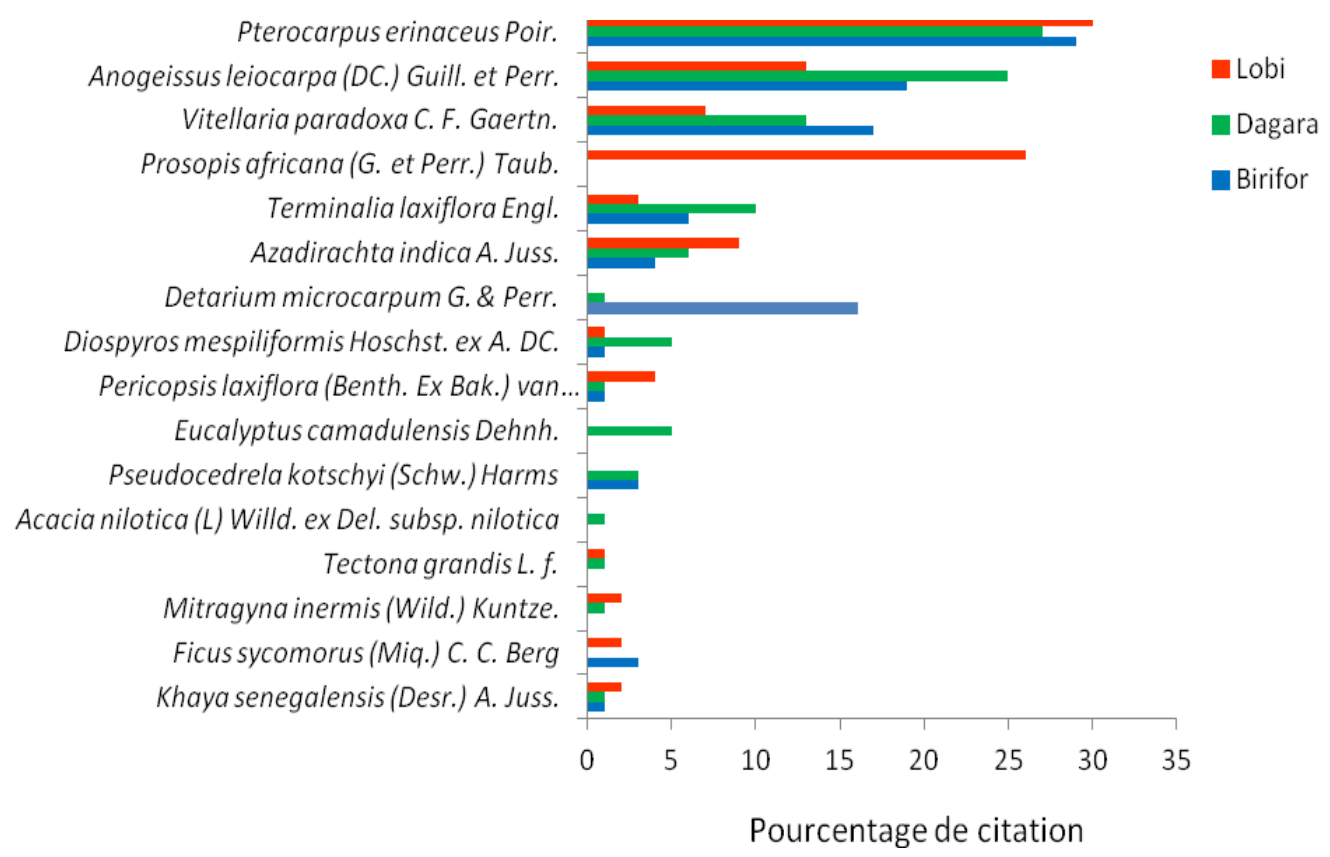

Figure 6 : Substituts de B. africana selon les groupes ethniques dans le Sud-Ouest du Burkina Faso. 


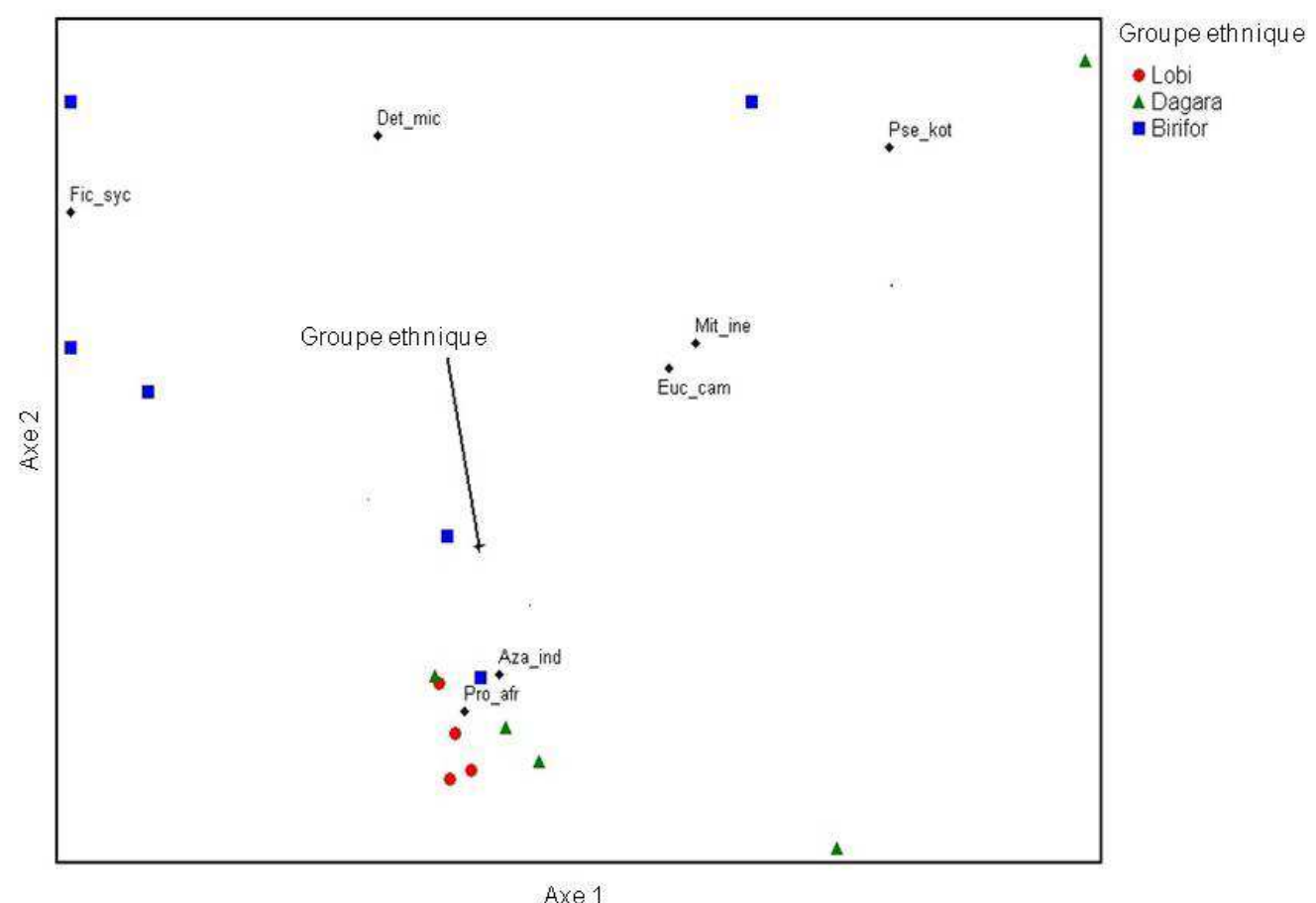

Figure 7: Ordination des substituts de B. africana. Ara_ind: Azadirachta indica, Det_mic: Detarium microcarpum, Euc_cam: Eucalyptus camadulensis, Fic_syc: Ficus sycomorus, Mit_ine: Mitragyna inermis, Pro_afr: Prosopis africana, Pse_kot: Pseudocedrela kotschyi.

Tableau 1: Substituts de Crateva adansonii en fonction de l'importance de citation, l'organe utilisé et le type de plante.

\begin{tabular}{rlcccc}
\hline Rang & Substitut & Organe utilisé & $\begin{array}{c}\text { Fréquence de } \\
\text { citation }(\boldsymbol{\%})\end{array}$ & $\begin{array}{c}\text { Plante cultivée } \\
\text { ou achetée }\end{array}$ & $\begin{array}{c}\text { Plante } \\
\text { locale }\end{array}$ \\
\hline 1 Moringa oleifera & feuilles & 26,5 & + & \\
2 & Vitex doniana & feuilles & 19 & & + \\
3 & Adansonia digitata & feuilles & 17,5 & & + \\
4 & Strychnos spinosa & feuilles & 9,3 & & + \\
5 & Balanites aegyptiaca & feuilles et fleurs & 6,9 & & + \\
6 & Pterocarpus santalinoides & feuilles & 4,1 & & + \\
7 & Ceiba pentandra & feuilles; & 3,6 & & + \\
8 & Légumes & feuilles et fruits & 2,8 & + & \\
9 & Ficus glumosa & feuilles & 2,1 & & + \\
10 & Ficus sycomorus & feuilles & 1 & & + \\
11 & Bombax costatum & fleurs & 0,8 & & + \\
12 & Hibiscus asper & feuilles & 0,8 & & + \\
13 & Maerua angolensis & feuilles & 0,8 & & + \\
14 & Cassia tora & feuilles & 0,5 & & + \\
15 & Ceratotheca sesamoides & feuilles & 0,5 & & + \\
16 & Leptadenia hastata & feuilles & 0,5 & & + \\
& Aucun substitut & & 3,3 & & \\
\hline
\end{tabular}


S. A. KABORE et al. /Int. J. Biol. Chem. Sci. 9(3): 1194-1208, 2015

Tableau 2: Substituts de Sarcocephalus latifolius en fonction de l'importance de citation, l'organe utilisé et le type de plante.

\begin{tabular}{|c|c|c|c|c|c|c|}
\hline Rang & Substituts & Organe utilisé & Fréquence de citation (\%) & Plante cultivée ou achetée & Plante locale & Autre produits \\
\hline 1 & Cassia sieberiana & racine & 26,1 & & + & \\
\hline 2 & Strychnos spinosa & racine & 8 & & + & \\
\hline 3 & Burkea africana & feuilles, écorce et racines & 7,6 & & + & \\
\hline 4 & Vernonia colorata & feuilles & 6,5 & & + & \\
\hline 5 & Pseudocedrala kotschyi & racines & 5,4 & & + & \\
\hline 6 & Produits pharmaceutiques & & 4,7 & & & + \\
\hline 7 & Terminalia laxiflora & racines et feuilles & 4,3 & & + & \\
\hline 8 & Detarium microcarpum & racines et fruits & 4 & & + & \\
\hline 9 & Dioscorea sp. & racines & 3,6 & & + & \\
\hline 10 & Securidaca longepedunculata & racines & 3,6 & & + & \\
\hline 11 & Flacourtia indica & racines & 2,9 & & + & \\
\hline 12 & Ziziphus mucronata & racines & 2,5 & & + & \\
\hline 13 & Ximenia americana & racines & 2,2 & & + & \\
\hline 14 & Zanthoxylum zanthoxyloides & racines & 1,4 & & + & \\
\hline 15 & Moringa oleifera & fruits & 1,4 & + & & \\
\hline 16 & Tamarindus indica & racines & 1,1 & & + & \\
\hline 17 & Médicaments traditionnelles & & 1,1 & & & + \\
\hline 18 & Crateva adansonii & feuilles et racines & 1,1 & & + & \\
\hline 19 & Annona senegalensis & racines & 1,1 & & + & \\
\hline 20 & Mangifera indica & écorce & 0,7 & + & & \\
\hline 21 & Diospyros mespiliformis & fruits immature & 0,7 & & + & \\
\hline \multirow[t]{2}{*}{22} & Balanites aegyptiaca & fruits & 0,7 & & + & \\
\hline & Aucun substitut & & 9,1 & & & \\
\hline
\end{tabular}


Tableau 3: Substituts de Burkea africana en fonction de l'importance de citation, l'organe utilisé et le type de plante.

\begin{tabular}{lccccc}
\hline Rang & Substitut & $\begin{array}{c}\text { Partie } \\
\text { utilisée }\end{array}$ & $\begin{array}{c}\text { Fréquence de } \\
\text { citation }(\%)\end{array}$ & $\begin{array}{c}\text { Plante } \\
\text { cultivée ou } \\
\text { achetée }\end{array}$ & Plante locale \\
\hline 1 & Pterocarpus erinaceus & bois & 27,9 & & + \\
2 & Anogeissus leiocarpa & bois & 19,4 & & + \\
3 & Vitellaria paradoxa & bois & 12,7 & & + \\
4 & Prosopis africana & bois & 7,5 & & + \\
5 & Terminalia laxiflora & bois & 6,7 & & + \\
6 & Azadirachta indica & bois & 5,9 & + & + \\
7 & Detarium microcarpum & bois & 4,9 & & + \\
8 & Diospyros mespiliformis & bois & 2,6 & & + \\
9 & Pseudocedrela kotschyi & bois & 2,1 & & + \\
10 & Eucalyptus camadulensis & bois & 1,8 & + & + \\
11 & Pericopsis laxiflora & bois & 1,6 & & + \\
12 & Khaya senegalensis & bois & 1 & & + \\
13 & Ficus sycomorus & bois & 0,8 & & + \\
14 & Mitragyna inermis & bois & 0,8 & & + \\
15 & Tectona grandis & bois & 0,8 & + & + \\
16 & Acacia nilotica & bois & 0,5 & & + \\
& Aucun substitut & & 3,1 & & + \\
\hline
\end{tabular}

\section{DISCUSSION}

Moringa oleifera, le plus cité des substituts de C. adansonii, est une plante exotique originaire de l'Inde. Elle fait l'objet d'une promotion par les services en charge de l'environnement. C'est une espèce qui pourrait se substituer complètement à $C$. adansonii au vu de l'engouement qu'elle suscite au sein de la population enquêtée. Elle est plantée dans les parcs agroforestiers, avec des densités qui avoisinent 5 individus/ha dans certains villages. Vitex doniana (second substitut de $C$. adansonii) n'est pas une espèce très abondante dans la région. Quant à Strychnos spinosa, elle est relativement plus fréquente que $C$. adansonii mais elle subit de forte pression au regard de ses diverses utilisations.

A première vue, la récolte des feuilles porte moins de préjudice aux plantes par rapport à d'autres organes comme les racines ou les écorces, mais peut compromettre leur saine croissance quand elle n'est pas modérée. Des techniques de récolte peu recommandables comme l'abattage des arbres rapporté par Hahn-Hadjali et Thiombiano (2000), ou l'ébranchage total, sont à déplorer également pour certains substituts.

Cassia sieberiana est reconnu comme étant le meilleur substitut de $S$. latifolius par les trois groupes ethniques. C'est une espèce fréquente dans la zone d'étude. Son utilisation contre les maux de ventre corrobore les résultats de Traoré et al. (2013) qui ont rapporté son usage contre les parasites gastrointestinaux dans différentes localités du Burkina Faso.

La plupart des substituts des racines de S. latifolius sont malheureusement des racines d'autres plantes. Deux des substituts, Zanthoxylum zanthoxyloides (Thiombiano et al., 2010) et Securidaca longepedunculata (Hahn-Hadjali et Thiombiano, 2000; Belem et 
al., 2007; Thiombiano et al., 2010), sont menacés dans plusieurs localités du pays.

Pour le cas particulier des espèces médicinales comme $S$. latifolius, la substitution des racines par les feuilles est une approche qui conserve mieux la plante. Les travaux de Badiaga (2011) au Mali ont montré que les extraits de feuilles et celles de racines de $S$. latifolius donnent tous les mêmes niveaux d'activités analgésiques. Ces résultats montrent bien qu'il est tout à fait possible de substituer ses racines par ses feuilles pour le traitement des maux de ventre.

Dans d'autres pays, les études ont aussi montré que la substitution de certains organes par d'autres est une bonne mesure de conservation des espèces médicinales (Zschocke et al., 2000; Goussanou et al., 2013). Toutefois, comme l'ont déjà relevé Monteiro et al. (2006), l'utilisation des substituts médicinaux requiert une analyse phytochimique et pharmacologique afin d'évaluer leur efficacité et donc de confirmer leur interchangeabilité.

Dans la même logique, Diatta et al. (2014) ont démontré la possibilité de substituer les racines de Zanthoxylum zanthoxyloides par ses feuilles dans les soins de plusieurs pathologies. Un travail de vulgarisation des résultats de recherches doit donc être entrepris par les décideurs politiques pour mettre à la disposition du monde rurale les meilleurs pratiques qui contribuent à sauvegarder les ressources végétales.

Quant à $B$. africana, les résultats ont montré que Ptereoscarpus erinaceus est le premier substitut de son bois dans la construction. Cependant, $P$. erinaceus est une espèce protégée et est donc interdite de couper (Zida et al., 2009). En outre, elle est déjà menacée dans la zone sud soudanienne du Burkina Faso (Thiombiano et al., 2010). Les espèces plantées telles que Azadirachta indica et Tectona grandis pourront faire l'objet de vulgarisation à petite échelle afin de réduire la pression sur les formations naturelles. A. indica est même devenu le premier choix des populations pour la construction des maisons dans le centre du Burkina Faso (Yaméogo et al., 2013). Cependant, l'utilisation de certaines espèces exotiques comme l'eucalyptus devrait être encadrée car présentant des risques écologiques avérés comme la toxicité ou l'épuisement des nappes phréatiques (Liu et $\mathrm{Li}$, 2010). De façon durable, les politiques publiques en matière de gestion des forêts devraient aider les producteurs dans l'acquisition de paquets technologiques pour la mise en place de programmes de plantation des espèces locales très utilisées.

L'utilisation de Vitellaria paradoxa comme substituts au bois de B. africana, n'est pas acceptable car elle est non seulement interdite de couper (Zida et al., 2009), mais c'est également une espèce d'importance économique capitale pour le pays (INSD, 2009). Toute utilisation massive de cette plante autre que la récolte de fruits doit attirer l'attention des décideurs et des services en charges de l'environnement.

Par conservatisme sans doute, une faible proportion des enquêtés n'ont pas trouvé de substitut à l'une au moins des espèces. On peut alors malheureusement penser que ces personnes continueront d'exploiter ces espèces quel que que soit leur niveau de rareté.

Les résultats de l'étude montrent que le choix des substituts dépend plus du groupe ethnique. Heubach et al. (2013) signalaient aussi que l'affiliation aux groupes ethniques est un important facteur de différenciation des connaissances des usages des espèces. Il est donc judicieux de tenir compte de cette différence de point de vue selon les groupes ethniques dans l'orientation des actions de préservation. Comme l'ont noté Heubach et al. (2013), les savoirs traditionnels peuvent aussi varier suivant les conditions écologiques et l'abondance des espèces. L'utilisation des espèces de remplacement peut être vu comme un savoir propre au groupe ethnique, et relevant de l'identité culturelle du groupe.

Les populations optent très peu pour une substitution des espèces par des produits manufacturés. Elles trouvent par exemple 
qu'elles ne peuvent pas abandonner l'utilisation des plantes médicinales au profit des médicaments pharmaceutiques, par manque de moyens financiers. Le pouvoir d'achat des populations est donc un déterminant majeur de la préservation de la diversité végétale.

Il en est de même de l'utilisation du bois pour construire les toitures des maisons. Les matériaux comme les tôles et le ciment ne peuvent pas remplacer le bois dans l'édification des logements traditionnels. Utiliser ces matériaux reviendrait à changer de type d'habitation, et cela signera inéluctablement le début de l'abandon d'un pan important de l'identité culturelle de ces populations.

\section{Conclusion}

L'étude a permis de déterminer les substituts de $C$. adansonii, $S$. latifolius et $B$. africana au sein des groupes ethniques "Lobi", "Dagara" et "Birifor" du Burkina Faso. La grande majorité de ces substituts sont des espèces ligneuses locales. La baisse de fourniture des services écosystémiques fournis par ces espèces va se traduire par un changement d'utilisation d'espèce plutôt que par l'adoption de produits manufacturés qui jouent les mêmes rôles que ces plantes. L'étude a pu montrer que le choix des substituts dépend plus de la variable groupe ethnique. Il faudrait donc promouvoir un partage de connaissances entre ces groupes éthiques qui vivent dans la même région afin de fédérer leurs savoirs. Un changement d'attitude est nécessaire en faveur de l'adoption des plantations des espèces utiles plutôt que de continuer à s'adonner à la cueillette dans les formations naturelles. L'amélioration des revenus et l'éradication de la pauvreté monétaire des populations pourront contribuer à changer les stratégies de substitution des espèces par d'autres espèces. Les travaux sur les possibilités de substitution des plantes les plus utilisées et les plus menacées devront être poursuivis afin de documenter les tendances de changement d'utilisations des espèces. Les résultats issus de ces études serviront d'outils de veille pour la conservation des formations naturelles.

\section{REMERCIEMENTS}

Le travail a été réalisé dans le cadre du projet UNDESERT (Understanding and combating desertification to mitigate its impact on ecosystem services - European Union FP7 project no. 243906). Merci à toute l'équipe de UNDESERT en particulier Pr Irénée Somda, Dr Ilboudo Jean-Baptiste, Dr Dénis Ouédraogo, Dr Ouoba Paulin et Dr Jérôme Yaméogo pour leurs appuis multiformes. Les auteurs adressent également leurs remerciements à l'ensemble des personnes qui ont bien voulu participer à l'étude.

\section{REFERENCES}

Amos A, Abbah J, Chindo B, Edmond I, Binda L, Adzu B, Buhari S, Odutola AA, Wambebe C, Gamaniel K. 2005. Neuropharmacological effects of the aqueous extract of Nauclea latifolia root bark in rats and mice. $J$. Ethnopharmacol., 97: 53-57.

Arbonnier M. 2009. Arbres, Arbustes et Lianes d'Afrique de l'Ouest ( $3^{\text {ième }}$ edn). CIRAD-MNHN-UICN : Paris ; 573.

Bayala J, Ouédraogo SJ, Ong K. 2009. Early growth performance and water use of planted West African provenance of Vitellaria paradoxa C. F. Gaertn (karité) in Gonsé, Burkina Faso. Agroforest. Syst., 75: 117-127.

Belem B, Nacoulma BMI, Gbangou R, Kambou S, Hansen HH, Gausset Q, Lund S, Ræbild R, Lompo D, Ouédraogo M, Theilade I, Boussim IJ. 2007. Use of non-wood forest products by local people bordering the "Parc National Kaboré Tambi”, Burkina Faso. J. Transdiscip. Environ. Stud., 6(1): 121.

Boumendjel A, Taïwe GS, Bum EN, Chabrol T, Beney C, Sinniger V, Haudecoeur R, Marcourt L, Challal S, Queiroz EF, 
Souard F, Le Borgne M, Lomberget T, Depaulis A, Lavaud C, Robins R, Wolfender J-L, Bonaz B, De Waard M. 2013. Occurrence of the synthetic analgesic Tramadol in an african medicinal plant. Angew. Chem. Int. Ed., 52 : 11780-11784.

Diatta W, Sy GY, Manga CI, Diatta K, Fall AD, Bassene E. 2014. Recherche des activités anti-inflammatoire et analgésique des extraits de feuilles de Zanthoxylum zanthoxyloides (Lam) zepernick et timler (Rutaceae). Int. J. Biol. Chem. Sci., 8(1) : 128-133.

Dobson A, Lodge D, Alder J, Cumming GS, Keymer J, McGlade J, Mooney $\mathrm{H}$, Rusak JA, Sala O, Wolters V, Wall D, Winfree R, Xenopoulos MA. 2006. Habitat loss, trophic collapse, and the decline of ecosystem services. Ecology, 87: 1915-1924.

Fisher B, Turner RK, Morling P. 2009. Defining and classifying ecosystem services for decision making. Ecol. Econ., 68: 643-653.

Boussim JI. 2010. Phytogeographic territories. In Biodiversity Atlas of West Africa, Volume II : Burkina Faso. Thiombiano A, Kampmann D (eds). Frankurt/Main : Ouagadougou; 152-155.

Goussanou CA, Assogbadjo AE, Gouwakinnou GN, Glèlè-Kakaï RL, Chakeredza S, Sinsin B. 2013. Biomass, root structure and morphological characteristics of the medicinal Sarcocephalus latifolius (Sm) E.A. Bruce shrub across different ecologies in Benin. QScience Connect, 12: 1-9.

Hahn-Hadjali K, Thiombiano A. 2000. Perception des espèces en voie de disparition en milieu Gourmantché (Est du Burkina Faso). Berichte des Sonderforschungsbereichs, 268: 285297.

Heubach K, Wittig R, Nuppenau E-A, Hahn K. 2013. Local values, social differentiation and conservation efforts : the impact of ethnic affiliation on the valuation of NTFP-Species in Northern Benin, West Africa. Hum. Ecol., 41: 513-533.

Heubes J, Schmidt M, Stuch B, García Márquez JR, Wittig R, Zizka G, Thiombiano A, Sinsin B, Schaldach R, Hahn K. 2013. The projected impact of climate and land use change on plant diversity: An example from West Africa. Journal of Arid Environments, 96:48-54.

INSD (Institut National de la Statistique et de la Démographie). 2009. Annuaire Statistique. INSD : Burkina Faso ; 413.

Iwueke AV, Nwodo OFC. 2008. Antihyperglycaemic effect of aqueous extract of Daniella oliveri and Sarcocephalus latifolius roots on key carbohydrate metabolic enzymes and glycogen in experimental diabetes. Biokemistri, 20(2): 63-70.

Kaboré SA, Hien M, Ouédraogo D, Diallo TRE, Hahn K, Nacro HB. 2014. Use of ecosystem services of Sarcocephalus latifolius (Sm.) E.A. Bruce and induced effect of human pressure on the species in the Southwestern Region of Burkina Faso. Ethnobotany Research and Applications, 12: 561-570.

Konaté S, Linsenmair KE. 2010. Biological diversity of West Africa: importance, threats and valorization. In Biodiversity Atlas of West Africa, Volume II: Burkina Faso. Thiombiano A, Kampmann D (eds). Frankurt/Main : Ouagadougou; 14-75.

Limoges B. 2009. Biodiversité, services écologiques et bien-être humain. Le Naturaliste Canadien, 133: 15-19.

Liu H, Li J. 2010. The study of the ecological problems of Eucalyptus plantation and sustainable development in Maoming Xiaoliang. Journal of Sustainable Development, 3(1): 197-201.

Mawdsley J, O’Malley R, Ojima DS. 2006. A review of climate-change adaptation strategies for wildlife management and 
biodiversity conservation. Conserv. Biol., 23 (5): 1080-1089.

McCune B, Mefford MJ. 2011. PC-ORD. Multivariate Analysis of Ecological Data. Version 6.0 MjM Software. Gleneden Beach : Oregon, U.S.A.

Monteiro JM, Albuquerque UP, Lins Neto EMF, Araújo EL, Amorim ELC. 2006. Use patterns and knowledge of medicinal species among two rural communities in Brazil's Semi-Arid Northeastern Region. J. Ethnopharmacol., 105: 173-186.

Neya B, Hakkou M, Pétrissans M, Gérardin P. 2004. On the durability of Burkea africana heartwood: evidence of biocidal and hydrophobic properties responsible for durability. Ann. For. Sci., 61(3): 277282.

Badiaga M. 2011. Etude ethnobotanique, phytochimique, et activités biologique de Nauclea latifolia Smith, une plante médicinale récoltée au Mali. Thèse, Université de Bamako, Mali, 183 p.

Thiombiano A, Schmidt M, Da S, HahnHadjali K, Zizka G, Wittig R. 2010. Vascular plants : flowering plants. In Biodiversity atlas of West Africa, Volume II : Burkina Faso, Thiombiano A, Kampmann D (eds). Frankfurt/main: Ouagadougou; 184-192.

Traoré A, Ouedraogo S, Lompo M, Traoré S, Somé N, Guissou IP. 2013.
Ethnobotanical survey of medicinal plants used to treat gastrointestinal parasites in human and livestock in four geographic areas of Burkina Faso (West Africa). Arch. Appl. Sci. Res., 5(6) : 172177.

Yaméogo G, Yélémou B, Kaboré O, Traoré D. 2013. Bois d'énergie du 'dolo'et bois de service: deux modes de consommation de bois à Vipalogo au Burkina Faso. Bulletin de la Recherche Agronomique du Bénin, 73 : 1-12.

Yesufu HB, Bassi PU, Khan IZ, Abdulrahaman FI, Mohammed GT. 2010. Phytochemical screening and hepatoprotective properties of the aqueous root bark extract of Sarcocephalus latifolius (Smith) Bruce (African Peach). Acta Microbiologica, 1(2) : 1-5.

Zida D, Tigabu M, Sawadogo L, Tiveau D, Odén PC. 2009. Long-term effects of prescribed early fire, grazing and selective tree cutting on seedling populations in the Sudanian savanna of Burkina Faso. Afr. J. Ecol., 47: 97-108.

Zschocke S, Rabe T, Staden J. 2000. Plant part substitution-a way to conserve endangered medicinal plants? $J$. Ethnopharmacol., 71: 281-292. 